\title{
Norm inequalities of Čebyšev type for power series in Banach algebras
}

\author{
Silvestru S Dragomir ${ }^{1,2^{*}}$, Marius V Boldea ${ }^{3}$, Constantin Buşe $e^{4}$ and Mihail Megan ${ }^{4}$
}

"Correspondence:
sever.dragomir@vu.edu.au;
http://rgmia.org/dragomir
${ }^{1}$ Mathematics, School of
Engineering \& Science, Victoria
University, PO Box 14428,
Melbourne City, MC 8001, Australia
${ }^{2}$ School of Computational \&
Applied Mathematics, University of
the Witwatersrand, Private Bag 3,
Johannesburg, 2050, South Africa
Full list of author information is
available at the end of the article

available at the end of the article

\begin{abstract}
Let $f(\lambda)=\sum_{n=0}^{\infty} \alpha_{n} \lambda^{n}$ be a function defined by power series with complex coefficients and convergent on the open disk $D(0, R) \subset \mathbb{C}, R>0$ and $x, y \in \mathcal{B}$, a Banach algebra, with $x y=y x$. In this paper we establish some upper bounds for the norm of the Cebyšev type difference $f(\lambda) f(\lambda x y)-f(\lambda x) f(\lambda y)$, provided that the complex number $\lambda$ and the vectors $x, y \in \mathcal{B}$ are such that the series in the above expression are convergent. Applications for some fundamental functions such as the exponential function and the resolvent function are provided as well.
\end{abstract}

MSC: 47A63; 47A99

Keywords: Banach algebras; power series; exponential function; resolvent function; norm inequalities

\section{Introduction}

For two Lebesgue integrable functions $f, g:[a, b] \rightarrow \mathbb{R}$, consider the Čebyšev functional:

$$
C(f, g):=\frac{1}{b-a} \int_{a}^{b} f(t) g(t) d t-\frac{1}{(b-a)^{2}} \int_{a}^{b} f(t) d t \int_{a}^{b} g(t) d t .
$$

In 1935, Grüss [1] showed that

$$
|C(f, g)| \leq \frac{1}{4}(M-m)(N-n),
$$

provided that there exist real numbers $m, M, n, N$ such that

$$
m \leq f(t) \leq M \quad \text { and } \quad n \leq g(t) \leq N \quad \text { for a.e. } t \in[a, b] .
$$

The constant $\frac{1}{4}$ is best possible in (1.1) in the sense that it cannot be replaced by a smaller quantity.

Another, however, less known result, even though it was obtained by Čebyšev in 1882 [2], states that

$$
|C(f, g)| \leq \frac{1}{12}\left\|f^{\prime}\right\|_{\infty}\left\|g^{\prime}\right\|_{\infty}(b-a)^{2},
$$

provided that $f^{\prime}, g^{\prime}$ exist and are continuous on $[a, b]$ and $\left\|f^{\prime}\right\|_{\infty}=\sup _{t \in[a, b]}\left|f^{\prime}(t)\right|$. The constant $\frac{1}{12}$ cannot be improved in the general case.

(c) 2014 Dragomir et al.; licensee Springer. This is an Open Access article distributed under the terms of the Creative Commons Attribution License (http://creativecommons.org/licenses/by/2.0), which permits unrestricted use, distribution, and reproduction in any medium, provided the original work is properly cited. 
The Čebyšev inequality (1.4) also holds if $f, g:[a, b] \rightarrow \mathbb{R}$ are assumed to be absolutely continuous and $f^{\prime}, g^{\prime} \in L_{\infty}[a, b]$, while $\left\|f^{\prime}\right\|_{\infty}=\operatorname{ess}_{\sup } \operatorname{su}_{t \in[a, b]}\left|f^{\prime}(t)\right|$.

A mixture between Grüss' result (1.2) and Čebyšev's one (1.4) is the following inequality obtained by Ostrowski in 1970 [3]:

$$
|C(f, g)| \leq \frac{1}{8}(b-a)(M-m)\left\|g^{\prime}\right\|_{\infty}
$$

provided that $f$ is Lebesgue integrable and satisfies (1.3), while $g$ is absolutely continuous and $g^{\prime} \in L_{\infty}[a, b]$. The constant $\frac{1}{8}$ is best possible in (1.5).

The case of Euclidean norms of the derivative was considered by Lupaş in [4] in which he proved that

$$
|C(f, g)| \leq \frac{1}{\pi^{2}}\left\|f^{\prime}\right\|_{2}\left\|g^{\prime}\right\|_{2}(b-a),
$$

provided that $f, g$ are absolutely continuous and $f^{\prime}, g^{\prime} \in L_{2}[a, b]$. The constant $\frac{1}{\pi^{2}}$ is the best possible.

Recently, Cerone and Dragomir [5] have proved the following results:

$$
|C(f, g)| \leq \inf _{\gamma \in \mathbb{R}}\|g-\gamma\|_{q} \cdot \frac{1}{b-a}\left(\int_{a}^{b}\left|f(t)-\frac{1}{b-a} \int_{a}^{b} f(s) d s\right|^{p} d t\right)^{\frac{1}{p}}
$$

where $p>1$ and $\frac{1}{p}+\frac{1}{q}=1$ or $p=1$ and $q=\infty$, and

$$
|C(f, g)| \leq \inf _{\gamma \in \mathbb{R}}\|g-\gamma\|_{1} \cdot \frac{1}{b-a} \operatorname{ess} \sup _{t \in[a, b]}\left|f(t)-\frac{1}{b-a} \int_{a}^{b} f(s) d s\right|,
$$

provided that $f \in L_{p}[a, b]$ and $g \in L_{q}[a, b]\left(p>1, \frac{1}{p}+\frac{1}{q}=1 ; p=1, q=\infty\right.$ or $\left.p=\infty, q=1\right)$.

Notice that for $q=\infty, p=1$ in (1.7) we obtain

$$
\begin{aligned}
|C(f, g)| & \leq \inf _{\gamma \in \mathbb{R}}\|g-\gamma\|_{\infty} \cdot \frac{1}{b-a} \int_{a}^{b}\left|f(t)-\frac{1}{b-a} \int_{a}^{b} f(s) d s\right| d t \\
& \leq\|g\|_{\infty} \cdot \frac{1}{b-a} \int_{a}^{b}\left|f(t)-\frac{1}{b-a} \int_{a}^{b} f(s) d s\right| d t
\end{aligned}
$$

and if $g$ satisfies (1.3), then

$$
\begin{aligned}
|C(f, g)| & \leq \inf _{\gamma \in \mathbb{R}}\|g-\gamma\|_{\infty} \cdot \frac{1}{b-a} \int_{a}^{b}\left|f(t)-\frac{1}{b-a} \int_{a}^{b} f(s) d s\right| d t \\
& \leq\left\|g-\frac{n+N}{2}\right\|_{\infty} \cdot \frac{1}{b-a} \int_{a}^{b}\left|f(t)-\frac{1}{b-a} \int_{a}^{b} f(s) d s\right| d t \\
& \leq \frac{1}{2}(N-n) \cdot \frac{1}{b-a} \int_{a}^{b}\left|f(t)-\frac{1}{b-a} \int_{a}^{b} f(s) d s\right| d t .
\end{aligned}
$$

The inequality between the first and the last term in (1.10) has been obtained by Cheng and Sun in [6]. However, the sharpness of the constant $\frac{1}{2}$, a generalization for the abstract Lebesgue integral, and the discrete version of it have been obtained in [7]. 
For other recent results on the Grüss inequality, see [8-22], and the references therein. In order to consider a Čebyšev type functional for functions of vectors in Banach algebras, we need some preliminary definitions and results as follows.

\section{Some facts on Banach algebras}

Let $\mathcal{B}$ be an algebra. An algebra norm on $\mathcal{B}$ is a map $\|\cdot\|: \mathcal{B} \rightarrow[0, \infty)$ such that $(\mathcal{B},\|\cdot\|)$ is a normed space, and, further

$$
\|a b\| \leq\|a\|\|b\|
$$

for any $a, b \in \mathcal{B}$. The normed algebra $(\mathcal{B},\|\cdot\|)$ is a Banach algebra if $\|\cdot\|$ is a complete norm.

We assume that the Banach algebra is unital, this means that $\mathcal{B}$ has an identity 1 and that $\|1\|=1$.

Let $\mathcal{B}$ be a unital algebra. An element $a \in \mathcal{B}$ is invertible if there exists an element $b \in \mathcal{B}$ with $a b=b a=1$. The element $b$ is unique; it is called the inverse of $a$ and written $a^{-1}$ or $\frac{1}{a}$. The set of invertible elements of $\mathcal{B}$ is denoted by $\operatorname{Inv} \mathcal{B}$. If $a, b \in \operatorname{Inv} \mathcal{B}$ then $a b \in \operatorname{Inv} \mathcal{B}$ and $(a b)^{-1}=b^{-1} a^{-1}$.

For a unital Banach algebra we also have:

(i) if $a \in \mathcal{B}$ and $\lim _{n \rightarrow \infty}\left\|a^{n}\right\|^{1 / n}<1$, then $1-a \in \operatorname{Inv} \mathcal{B}$;

(ii) $\{a \in \mathcal{B}:\|1-b\|<1\} \subset \operatorname{Inv} \mathcal{B}$;

(iii) Inv $\mathcal{B}$ is an open subset of $\mathcal{B}$;

(iv) the map Inv $\mathcal{B} \ni a \longmapsto a^{-1} \in \operatorname{Inv} \mathcal{B}$ is continuous.

For simplicity, we denote $\lambda 1$, where $\lambda \in \mathbb{C}$ and 1 is the identity of $\mathcal{B}$, by $\lambda$. The resolvent set of $a \in \mathcal{B}$ is defined by

$$
\rho(a):=\{\lambda \in \mathbb{C}: \lambda-a \in \operatorname{Inv} \mathcal{B}\}
$$

the spectrum of $a$ is $\sigma(a)$, the complement of $\rho(a)$ in $\mathbb{C}$, and the resolvent function of $a$ is $R_{a}: \rho(a) \rightarrow \operatorname{Inv} \mathcal{B}, R_{a}(\lambda):=(\lambda-a)^{-1}$. For each $\lambda, \gamma \in \rho(a)$ we have the identity

$$
R_{a}(\gamma)-R_{a}(\lambda)=(\lambda-\gamma) R_{a}(\lambda) R_{a}(\gamma)
$$

We also have $\sigma(a) \subset\{\lambda \in \mathbb{C}:|\lambda| \leq\|a\|\}$. The spectral radius of $a$ is defined as $v(a)=$ $\sup \{|\lambda|: \lambda \in \sigma(a)\}$.

If $a, b$ are commuting elements in $\mathcal{B}$, i.e. $a b=b a$, then

$$
v(a b) \leq v(a) v(b) \quad \text { and } \quad v(a+b) \leq v(a)+v(b) .
$$

Let $\mathcal{B}$ a unital Banach algebra and $a \in \mathcal{B}$. Then

(i) the resolvent set $\rho(a)$ is open in $\mathbb{C}$;

(ii) for any bounded linear functionals $\lambda: \mathcal{B} \rightarrow \mathbb{C}$, the function $\lambda \circ R_{a}$ is analytic on $\rho(a)$;

(iii) the spectrum $\sigma(a)$ is compact and nonempty in $\mathbb{C}$;

(iv) for each $n \in \mathbb{N}$ and $r>v(a)$, we have

$$
a^{n}=\frac{1}{2 \pi i} \int_{|\xi|=r} \xi^{n}(\xi-a)^{-1} d \xi
$$

(v) we have $v(a)=\lim _{n \rightarrow \infty}\left\|a^{n}\right\|^{1 / n}$. 
Let $f$ be an analytic functions on the open disk $D(0, R)$ given by the power series $f(\lambda):=$ $\sum_{j=0}^{\infty} \alpha_{j} \lambda^{j}(|\lambda|<R)$. If $v(a)<R$, then the series $\sum_{j=0}^{\infty} \alpha_{j} a^{j}$ converges in the Banach algebra $\mathcal{B}$ because $\sum_{j=0}^{\infty}\left|\alpha_{j}\right|\left\|a^{j}\right\|<\infty$, and we can define $f(a)$ to be its sum. Clearly $f(a)$ is well defined and there are many examples of important functions on a Banach algebra $\mathcal{B}$ that can be constructed in this way. For instance, the exponential map on $\mathcal{B}$ denoted exp and defined as

$$
\exp a:=\sum_{j=0}^{\infty} \frac{1}{j !} a^{j} \quad \text { for each } a \in \mathcal{B} .
$$

If $\mathcal{B}$ is not commutative, then many of the familiar properties of the exponential function from the scalar case do not hold. The following key formula is valid, however, with the additional hypothesis of commutativity for $a$ and $b$ from $\mathcal{B}$ :

$$
\exp (a+b)=\exp (a) \exp (b)
$$

In a general Banach algebra $\mathcal{B}$ it is difficult to determine the elements in the range of the exponential map $\exp (\mathcal{B})$, i.e. the element which have a 'logarithm'. However, it is easy to see that if $a$ is an element in $B$ such that $\|1-a\|<1$, then $a$ is in $\exp (\mathcal{B})$. That follows from the fact that if we set

$$
b=-\sum_{n=1}^{\infty} \frac{1}{n}(1-a)^{n}
$$

then the series converges absolutely and, as in the scalar case, substituting this series into the series expansion for $\exp (b)$ yields $\exp (b)=a$.

It is well known that if $x$ and $y$ are commuting, i.e. $x y=y x$, then the exponential function satisfies the property

$$
\exp (x) \exp (y)=\exp (y) \exp (x)=\exp (x+y)
$$

Also, if $x$ is invertible and $a, b \in \mathbb{R}$ with $a<b$ then

$$
\int_{a}^{b} \exp (t x) d t=x^{-1}[\exp (b x)-\exp (a x)]
$$

Moreover, if $x$ and $y$ are commuting and $y-x$ is invertible, then

$$
\begin{aligned}
\int_{0}^{1} \exp ((1-s) x+s y) d s & =\int_{0}^{1} \exp (s(y-x)) \exp (x) d s \\
& =\left(\int_{0}^{1} \exp (s(y-x)) d s\right) \exp (x) \\
& =(y-x)^{-1}[\exp (y-x)-I] \exp (x) \\
& =(y-x)^{-1}[\exp (y)-\exp (x)] .
\end{aligned}
$$

Let $f(\lambda)=\sum_{n=0}^{\infty} \alpha_{n} \lambda^{n}$ be a function defined by power series with complex coefficients and convergent on the open $\operatorname{disk} D(0, R) \subset \mathbb{C}, R>0$ and $x, y \in \mathcal{B}$ with $x y=y x$. In this paper 
we establish some upper bounds for the norm of the Čebyšev type difference

$$
f(\lambda) f(\lambda x y)-f(\lambda x) f(\lambda y)
$$

provided that the complex number $\lambda$ and the vectors $x, y \in \mathcal{B}$ are such that the series in (2.1) are convergent. Applications for some fundamental functions such as the exponential function and the resolvent function are provided as well.

Inequalities for functions of operators in Hilbert spaces may be found in [23-26], the recent monographs [27-29], and the references therein.

\section{The results}

We denote by $\mathbb{C}$ the set of all complex numbers. Let $\alpha_{n}$ be nonzero complex numbers and let

$$
R:=\frac{1}{\limsup \left|\alpha_{n}\right|^{\frac{1}{n}}}
$$

Clearly $0 \leq R \leq \infty$, but we consider only the case $0<R \leq \infty$.

Denote by

$$
D(0, R)= \begin{cases}\{z \in \mathbb{C}:|z|<R\}, & \text { if } R<\infty, \\ \mathbb{C}, & \text { if } R=\infty,\end{cases}
$$

consider the functions

$$
\lambda \mapsto f(\lambda): D(0, R) \rightarrow \mathbb{C}, \quad f(\lambda):=\sum_{n=0}^{\infty} \alpha_{n} \lambda^{n}
$$

and

$$
\lambda \mapsto f_{A}(\lambda): D(0, R) \rightarrow \mathbb{C}, \quad f_{A}(\lambda):=\sum_{n=0}^{\infty}\left|\alpha_{n}\right| \lambda^{n}
$$

Let $\mathcal{B}$ be a unital Banach algebra and 1 its unity. Denote by

$$
B(0, R)= \begin{cases}\{x \in \mathcal{B}:\|x\|<R\}, & \text { if } R<\infty \\ \mathcal{B}, & \text { if } R=\infty\end{cases}
$$

We associate to $f$ the map

$$
x \mapsto \tilde{f}(x): B(0, R) \rightarrow \mathcal{B}, \quad \tilde{f}(x):=\sum_{n=0}^{\infty} \alpha_{n} x^{n} .
$$

Obviously, $\tilde{f}$ is correctly defined because the series $\sum_{n=0}^{\infty} \alpha_{n} x^{n}$ is absolutely convergent, since $\sum_{n=0}^{\infty}\left\|\alpha_{n} x^{n}\right\| \leq \sum_{n=0}^{\infty}\left|\alpha_{n}\right|\|x\|^{n}$.

In addition, we assume that $s_{2}:=\sum_{n=0}^{\infty} n^{2}\left|\alpha_{n}\right|<\infty$. Let $s_{0}:=\sum_{n=0}^{\infty}\left|\alpha_{n}\right|<\infty$ and $s_{1}:=$ $\sum_{n=0}^{\infty} n\left|\alpha_{n}\right|<\infty$. 
With the above assumptions we have the following.

Theorem 1 Let $\lambda \in \mathbb{C}$ such that $\max \left\{|\lambda|,|\lambda|^{2}\right\}<R<\infty$ and let $x, y \in \mathcal{B}$ with $\|x\|,\|y\| \leq 1$, and $x y=y x$. Then:

(i) We have

$$
\begin{aligned}
& \|\tilde{f}(\lambda \cdot 1) \tilde{f}(\lambda x y)-\tilde{f}(\lambda x) \tilde{f}(\lambda y)\| \\
& \quad \leq \sqrt{2} \psi \min \{\|x-1\|,\|y-1\|\} f_{A}\left(|\lambda|^{2}\right)
\end{aligned}
$$

where

$$
\psi^{2}:=s_{0} s_{2}-s_{1}^{2}
$$

(ii) We also have

$$
\begin{aligned}
\|\tilde{f}(\lambda \cdot 1) \tilde{f}(\lambda x y)-\tilde{f}(\lambda x) \tilde{f}(\lambda y)\| \\
\leq \sqrt{2} \min \{\|x-1\|,\|y-1\|\} f_{A}(|\lambda|) \\
\quad \times\left\{f_{A}(|\lambda|)\left[|\lambda| f_{A}^{\prime}(|\lambda|)+|\lambda|^{2} f_{A}^{\prime \prime}(|\lambda|)\right]-\left[|\lambda| f_{A}^{\prime}(|\lambda|)\right]^{2}\right\}^{1 / 2} .
\end{aligned}
$$

Proof For $m \geq 1$ and since $x y=y x$ we have

$$
\begin{aligned}
& \sum_{n=0}^{m} \sum_{j=0}^{m} \alpha_{n} \alpha_{j} \lambda^{n} \lambda^{j}\left(x^{n}-x^{j}\right) y^{n} \\
& \quad=\sum_{n=0}^{m} \sum_{j=0}^{m} \alpha_{n} \alpha_{j} \lambda^{n} \lambda^{j} x^{n} y^{n}-\sum_{n=0}^{m} \sum_{j=0}^{m} \alpha_{n} \alpha_{j} \lambda^{n} \lambda^{j} x^{j} y^{n} \\
& =\sum_{j=0}^{m} \alpha_{j} \lambda^{j} \sum_{n=0}^{m} \alpha_{n} \lambda^{n} x^{n} y^{n}-\sum_{j=0}^{m} \alpha_{j} \lambda^{j} x^{j} \sum_{n=0}^{m} \alpha_{n} \lambda^{n} y^{n} \\
& =\sum_{j=0}^{m} \alpha_{j} \lambda^{j} \sum_{n=0}^{m} \alpha_{n} \lambda^{n}(x y)^{n}-\sum_{j=0}^{m} \alpha_{j} \lambda^{j} x^{j} \sum_{n=0}^{m} \alpha_{n} \lambda^{n} y^{n}
\end{aligned}
$$

for any $\lambda \in \mathbb{C}$.

Taking the norm in (3.4) we have

$$
\begin{gathered}
\left\|\sum_{j=0}^{m} \alpha_{j} \lambda^{j} \sum_{n=0}^{m} \alpha_{n} \lambda^{n}(x y)^{n}-\sum_{j=0}^{m} \alpha_{j} \lambda^{j} x^{j} \sum_{n=0}^{m} \alpha_{n} \lambda^{n} y^{n}\right\| \\
\leq \sum_{n=0}^{m} \sum_{j=0}^{m}\left|\alpha_{n}\left\|\left.\alpha_{j}|| \lambda\right|^{n}|\lambda|^{j}\right\|\left(x^{n}-x^{j}\right) y^{n} \|\right. \\
\leq \sum_{n=0}^{m} \sum_{j=0}^{m}\left|\alpha_{n}\left\|\left.\alpha_{j}|| \lambda\right|^{n}|\lambda|^{j}\right\| x^{n}-x^{j}\|\| y^{n} \|\right. \\
\leq \sum_{n=0}^{m} \sum_{j=0}^{m}\left|\alpha_{n}\left\|\left.\alpha_{j}|| \lambda\right|^{n}|\lambda|^{j}\right\| x^{n}-x^{j}\|\| y \|^{n}\right.
\end{gathered}
$$




$$
\begin{aligned}
& \leq \sum_{n=0}^{m} \sum_{j=0}^{m}\left|\alpha_{n}\right|\left|\alpha_{j}\right||\lambda|^{n}|\lambda|^{j}\left\|x^{n}-x^{j}\right\| \\
& =2 \sum_{0 \leq j<n \leq m}\left|\alpha_{n}\right|\left|\alpha_{j}\right||\lambda|^{n}|\lambda|^{j}\left\|x^{n}-x^{j}\right\|,
\end{aligned}
$$

for any $\lambda \in \mathbb{C}$ and $m \geq 1$.

Observe that

$$
\begin{aligned}
L & :=\sum_{0 \leq j<n \leq m}\left|\alpha_{n}\right|\left|\alpha_{j}\right||\lambda|^{n}|\lambda|^{j}\left\|x^{n}-x^{j}\right\| \\
& =\sum_{0 \leq j<n \leq m}\left|\alpha_{n}\right|\left|\alpha_{j}\right||\lambda|^{n}|\lambda|^{j}\left\|\sum_{\ell=j}^{n-1}\left(x^{\ell+1}-x^{\ell}\right)\right\| \\
& =\sum_{0 \leq j<n \leq m}\left|\alpha_{n}\right|\left|\alpha_{j}\right||\lambda|^{n}|\lambda|^{j}\left\|\sum_{\ell=j}^{n-1} x^{\ell}(x-1)\right\| \\
& \leq\|x-1\| \sum_{0 \leq j<n \leq m}\left|\alpha_{n}\right|\left|\alpha_{j}\right||\lambda|^{n}|\lambda|^{j} \sum_{\ell=j}^{n-1}\|x\|^{\ell}
\end{aligned}
$$

for any $\lambda \in \mathbb{C}$ and $m \geq 1$.

We have

$$
\sum_{\ell=j}^{n-1}\|x\|^{\ell} \leq(n-j) \max _{\ell \in\{j, \ldots, n-1\}}\|x\|^{\ell} \leq(n-j) \max _{\ell \in\{1, \ldots, m-1\}}\|x\|^{\ell}
$$

and then

$$
L \leq\|x-1\| \max _{\ell \in\{1, \ldots, m-1\}}\|x\|^{\ell} \sum_{0 \leq j<n \leq m}\left|\alpha_{n}\right|\left|\alpha_{j}\right||\lambda|^{n}|\lambda|^{j}(n-j) .
$$

From the first inequality in (3.7) and since $\|x\|<1$ we have

$$
\begin{aligned}
& \left\|\sum_{j=0}^{m} \alpha_{j} \lambda^{j} \sum_{n=0}^{m} \alpha_{n} \lambda^{n}(x y)^{n}-\sum_{j=0}^{m} \alpha_{j} \lambda^{j} x^{j} \sum_{n=0}^{m} \alpha_{n} \lambda^{n} y^{n}\right\| \\
& \leq 2\|x-1\| \sum_{0 \leq j<n \leq m}\left|\alpha_{n}\right|\left|\alpha_{j}\right||\lambda|^{n}|\lambda|^{j}(n-j) \\
& \quad=\|x-1\| \sum_{n=0}^{m} \sum_{j=0}^{m}\left|\alpha_{n}\right|\left|\alpha_{j}\right||\lambda|^{n}|\lambda|^{j}|n-j| .
\end{aligned}
$$

(i) Using the Cauchy-Bunyakovsky-Schwarz inequality for double sums,

$$
\sum_{n=0}^{m} \sum_{j=0}^{m} p_{n, j} a_{n, j} b_{n, j} \leq\left(\sum_{n=0}^{m} \sum_{j=0}^{m} p_{n, j} a_{n, j}^{2}\right)^{1 / 2}\left(\sum_{n=0}^{m} \sum_{j=0}^{m} p_{n, j} b_{n, j}^{2}\right)^{1 / 2},
$$


where $p_{n, j}, a_{n, j}, b_{n, j} \geq 0$ for $n, j \in\{0, \ldots, m\}$, we have

$$
\begin{aligned}
& \sum_{n=0}^{m} \sum_{j=0}^{m}\left|\alpha_{n}\right|\left|\alpha_{j}\right||\lambda|^{n}|\lambda|^{j}|n-j| \\
& \quad \leq\left(\sum_{n=0}^{m} \sum_{j=0}^{m}\left|\alpha_{n}\right|\left|\alpha_{j}\right||\lambda|^{2 n}|\lambda|^{2 j}\right)^{1 / 2}\left(\sum_{n=0}^{m} \sum_{j=0}^{m}\left|\alpha_{n}\right|\left|\alpha_{j}\right||n-j|^{2}\right)^{1 / 2} \\
& \quad=\sqrt{2}\left(\sum_{n=0}^{m}\left|\alpha_{n}\right||\lambda|^{2 n}\right)\left[\sum_{n=0}^{m}\left|\alpha_{n}\right| \sum_{n=0}^{m} n^{2}\left|\alpha_{n}\right|-\left(\sum_{n=0}^{m} n\left|\alpha_{n}\right|\right)^{2}\right]^{1 / 2}
\end{aligned}
$$

for any $\lambda \in \mathbb{C}$ and $m \geq 1$.

From (3.8) and (3.9) we get the inequality

$$
\begin{aligned}
\left\|\sum_{j=0}^{m} \alpha_{j} \lambda^{j} \sum_{n=0}^{m} \alpha_{n} \lambda^{n}(x y)^{n}-\sum_{j=0}^{m} \alpha_{j} \lambda^{j} x^{j} \sum_{n=0}^{m} \alpha_{n} \lambda^{n} y^{n}\right\| \\
\leq \sqrt{2}\|x-1\|\left(\sum_{n=0}^{m}\left|\alpha_{n}\right||\lambda|^{2 n}\right) \\
\times\left[\sum_{n=0}^{m}\left|\alpha_{n}\right| \sum_{n=0}^{m} n^{2}\left|\alpha_{n}\right|-\left(\sum_{n=0}^{m} n\left|\alpha_{n}\right|\right)^{2}\right]^{1 / 2} .
\end{aligned}
$$

Since the series

$$
\sum_{j=0}^{\infty} \alpha_{j} \lambda^{j}, \sum_{n=0}^{\infty} \alpha_{n} \lambda^{n}(x y)^{n}, \sum_{j=0}^{\infty} \alpha_{j} \lambda^{j} x^{j}, \sum_{n=0}^{\infty} \alpha_{n} \lambda^{n} y^{n}
$$

are convergent in $\mathcal{B}, \sum_{n=0}^{\infty}\left|\alpha_{n}\right||\lambda|^{2 n}$ is convergent and the limit

$$
\lim _{m \rightarrow \infty}\left[\sum_{n=0}^{m}\left|\alpha_{n}\right| \sum_{n=0}^{m} n^{2}\left|\alpha_{n}\right|-\left(\sum_{n=0}^{m} n\left|\alpha_{n}\right|\right)^{2}\right]^{1 / 2}
$$

exists, then by letting $m \rightarrow \infty$ in (3.10) we deduce the desired result in (3.1) for $x$. Due to the commutativity of $x$ with $y$, a similar result can be stated for $y$, and taking the minimum, we deduce the desired result.

(ii) Using the Cauchy-Bunyakovsky-Schwarz inequality for double sums,

$$
\sum_{n=0}^{m} \sum_{j=0}^{m} p_{n, j} a_{n, j} \leq\left(\sum_{n=0}^{m} \sum_{j=0}^{m} p_{n, j}\right)^{1 / 2}\left(\sum_{n=0}^{m} \sum_{j=0}^{m} p_{n, j} a_{n, j}^{2}\right)^{1 / 2}
$$

where $p_{n, j}, a_{n, j} \geq 0$ for $n, j \in\{0, \ldots, m\}$, we also have

$$
\begin{aligned}
& \sum_{n=0}^{m} \sum_{j=0}^{m}\left|\alpha_{n}\right|\left|\alpha_{j}\right||\lambda|^{n}|\lambda|^{j}|n-j| \\
& \quad \leq\left(\sum_{n=0}^{m} \sum_{j=0}^{m}\left|\alpha_{n}\right|\left|\alpha_{j}\right||\lambda|^{n}|\lambda|^{j}\right)^{1 / 2}\left(\sum_{n=0}^{m} \sum_{j=0}^{m}\left|\alpha_{n}\right|\left|\alpha_{j}\right||\lambda|^{n}|\lambda|^{j}|n-j|^{2}\right)^{1 / 2}
\end{aligned}
$$


for any $\lambda \in \mathbb{C}$ and $m \geq 1$.

From (3.8) and (3.11) we have

$$
\begin{aligned}
\left\|\sum_{j=0}^{m} \alpha_{j} \lambda^{j} \sum_{n=0}^{m} \alpha_{n} \lambda^{n}(x y)^{n}-\sum_{j=0}^{m} \alpha_{j} \lambda^{j} x^{j} \sum_{n=0}^{m} \alpha_{n} \lambda^{n} y^{n}\right\| \\
\leq \sqrt{2}\|x-1\|\left(\sum_{n=0}^{m}\left|\alpha_{n}\right||\lambda|^{n}\right) \\
\times\left[\sum_{n=0}^{m}\left|\alpha_{n}\right||\lambda|^{n} \sum_{n=0}^{m} n^{2}\left|\alpha_{n}\right||\lambda|^{n}-\left(\sum_{n=0}^{m} n\left|\alpha_{n}\right||\lambda|^{n}\right)^{2}\right]^{1 / 2}
\end{aligned}
$$

for any $\lambda \in \mathbb{C}$ and $m \geq 1$.

If we denote $f(u):=\sum_{n=0}^{\infty} \alpha_{n} u^{n}$, then for $|u|<R$ we have

$$
\sum_{n=0}^{\infty} n \alpha_{n} u^{n}=u f^{\prime}(u)
$$

and

$$
\sum_{n=0}^{\infty} n^{2} \alpha_{n} u^{n}=u\left(u g^{\prime}(u)\right)^{\prime}
$$

However

$$
u\left(u g^{\prime}(u)\right)^{\prime}=u g^{\prime}(u)+u^{2} g^{\prime \prime}(u)
$$

and then

$$
\sum_{n=0}^{\infty} n^{2} \alpha_{n} u^{n}=u g^{\prime}(u)+u^{2} g^{\prime \prime}(u) .
$$

Therefore

$$
\sum_{n=0}^{\infty} n^{2}\left|\alpha_{n}\right||\lambda|^{n}=|\lambda| f_{A}^{\prime}(|\lambda|)+|\lambda|^{2} f_{A}^{\prime \prime}(|\lambda|)
$$

and

$$
\sum_{n=0}^{m} n\left|\alpha_{n}\right||\lambda|^{n}=|\lambda| f^{\prime}(|\lambda|)
$$

for $|\lambda|<R$. 
Since all the series whose partial sums are involved in (3.12) are convergent, then by letting $m \rightarrow \infty$ in (3.12) we deduce the desired inequality (3.3) for $x$. Due to the commutativity of $x$ with $y$, a similar result can be stated for $y$, and taking the minimum, we deduce the desired result.

Remark 1 If $R=\infty$, Theorem 1 holds true. Moreover, in this case the restrictions $\|x\|,\|y\| \leq 1$ need no longer be imposed.

Remark 2 We observe that if the power series $f(\lambda)=\sum_{n=0}^{\infty} \alpha_{n} \lambda^{n}$ has the radius of convergence $R>1$, then

$$
\sum_{n=0}^{\infty}\left|\alpha_{n}\right|=f_{A}(1), \quad \sum_{n=0}^{\infty} n^{2}\left|\alpha_{n}\right|=f_{A}^{\prime}(1)+f_{A}^{\prime \prime}(1)
$$

and

$$
\sum_{n=0}^{\infty} n\left|\alpha_{n}\right|=f_{A}^{\prime}(1)
$$

In this case $\psi$ is finite and

$$
\begin{aligned}
\psi & =\lim _{m \rightarrow \infty}\left[\sum_{n=0}^{m}\left|\alpha_{n}\right| \sum_{n=0}^{m} n^{2}\left|\alpha_{n}\right|-\left(\sum_{n=0}^{m} n\left|\alpha_{n}\right|\right)^{2}\right]^{1 / 2} \\
& =\left\{f_{A}(1)\left[f_{A}^{\prime}(1)+f_{A}^{\prime \prime}(1)\right]-\left[f_{A}^{\prime}(1)\right]^{2}\right\}^{1 / 2} .
\end{aligned}
$$

Therefore, if $\lambda \in \mathbb{C}$ with $|\lambda|,|\lambda|^{2},|\lambda|\|x\|,|\lambda|\|y\|<R$, then from (3.1) we have

$$
\begin{aligned}
\|\tilde{f}(\lambda \cdot 1) \tilde{f}(\lambda x y)-\tilde{f}(\lambda x) \tilde{f}(\lambda y)\| \\
\leq \sqrt{2}\left\{f_{A}(1)\left[f_{A}^{\prime}(1)+f_{A}^{\prime \prime}(1)\right]-\left[f_{A}^{\prime}(1)\right]^{2}\right\}^{1 / 2} \\
\quad \times \min \{\|x-1\|,\|y-1\|\} f_{A}\left(|\lambda|^{2}\right) .
\end{aligned}
$$

Corollary 1 Under the assumptions of Theorem 1 we have the inequalities

$$
\left\|\tilde{f}(\lambda \cdot 1) \tilde{f}\left(\lambda x^{2}\right)-\tilde{f}^{2}(\lambda x)\right\| \leq \sqrt{2} \psi\|x-1\| f_{A}\left(|\lambda|^{2}\right)
$$

provided $\lambda \in \mathbb{C}$ with $|\lambda|,|\lambda|^{2},|\lambda|\|x\|<R$, and

$$
\begin{aligned}
& \| \tilde{f}(\lambda\cdot 1) \tilde{f}\left(\lambda x^{2}\right)-f^{2}(\lambda x) \| \\
& \leq \sqrt{2}\|x-1\| f_{A}(|\lambda|) \\
& \quad \times\left\{f_{A}(|\lambda|)\left[|\lambda| f_{A}^{\prime}(|\lambda|)+|\lambda|^{2} f_{A}^{\prime \prime}(|\lambda|)\right]-\left[|\lambda| f_{A}^{\prime}(|\lambda|)\right]^{2}\right\}^{1 / 2}
\end{aligned}
$$

provided $\lambda \in \mathbb{C}$ with $|\lambda|,|\lambda|\|x\|<R$. 
Theorem 2 Let $f(\lambda)=\sum_{n=0}^{\infty} \alpha_{n} \lambda^{n}$ be a function defined by power series with complex coefficients and convergent on the open disk $D(0, R) \subset \mathbb{C}, R>0$, and $x, y \in \mathcal{B}$ with $x y=y x$ and $\|x\|,\|y\|<1$.

If $\lambda \in \mathbb{C}$ with $|\lambda|,|\lambda|\|x\|,|\lambda|\|y\|<R$, then

$$
\|\tilde{f}(\lambda \cdot 1) \tilde{f}(\lambda x y)-\tilde{f}(\lambda x) \tilde{f}(\lambda y)\| \leq \min \left\{\frac{\|x-1\|}{1-\|x\|}, \frac{\|y-1\|}{1-\|y\|}\right\}\left[f_{A}^{2}(|\lambda|)-f_{A^{2}}\left(|\lambda|^{2}\right)\right]
$$

where

$$
f_{A^{2}}(\lambda):=\sum_{n=0}^{\infty}\left|\alpha_{n}\right|^{2} \lambda^{n}
$$

has the radius of convergence $R^{2}$.

Proof As pointed out in (3.6), we have

$$
\begin{aligned}
L & \leq\|x-1\| \sum_{0 \leq j<n \leq m}\left|\alpha_{n}\right|\left|\alpha_{j}\right||\lambda|^{n}|\lambda|^{j} \sum_{\ell=j}^{n-1}\|x\|^{\ell} \\
& \leq\|x-1\| \sum_{\ell=0}^{m-1}\|x\|^{\ell} \sum_{0 \leq j<n \leq m}\left|\alpha_{n}\left\|\alpha_{j}\right\| \lambda\right|^{n}|\lambda|^{j}
\end{aligned}
$$

for any $\lambda \in \mathbb{C}$ and $m \geq 1$.

Denote

$$
K_{m}:=\sum_{0 \leq j<n \leq m}\left|\alpha_{n}\right|\left|\alpha_{j}\right||\lambda|^{n}|\lambda|^{j} .
$$

We obviously have

$$
\begin{aligned}
K_{m} & =\frac{1}{2}\left(\sum_{n, j=0}^{m}\left|\alpha_{n}\right|\left|\alpha_{j}\right||\lambda|^{n}|\lambda|^{j}-\sum_{n=0}^{m}\left|\alpha_{n}\right|^{2}|\lambda|^{2 n}\right) \\
& =\frac{1}{2}\left[\left(\sum_{n=0}^{m}\left|\alpha_{n}\right||\lambda|^{n}\right)^{2}-\sum_{n=0}^{m}\left|\alpha_{n}\right|^{2}|\lambda|^{2 n}\right] .
\end{aligned}
$$

From (3.8) and (3.18) we get the inequality

$$
\begin{gathered}
\left\|\sum_{j=0}^{m} \alpha_{j} \lambda^{j} \sum_{n=0}^{m} \alpha_{n} \lambda^{n}(x y)^{n}-\sum_{j=0}^{m} \alpha_{j} \lambda^{j} x^{j} \sum_{n=0}^{m} \alpha_{n} \lambda^{n} y^{n}\right\| \\
\leq\|x-1\| \sum_{\ell=0}^{m-1}\|x\|^{\ell} \\
\times\left[\left(\sum_{n=0}^{m}\left|\alpha_{n}\right||\lambda|^{n}\right)^{2}-\sum_{n=0}^{m}\left|\alpha_{n}\right|^{2}|\lambda|^{2 n}\right]
\end{gathered}
$$

for any $\lambda \in \mathbb{C}$ and $m \geq 1$. 
Since all the series whose partial sums are involved in (3.19) are convergent, then by letting $m \rightarrow \infty$ in (3.19) we deduce the desired inequality (3.16) for $x$. Due to the commutativity of $x$ with $y$, a similar result can be stated for $y$, and taking the minimum, we deduce the desired result.

Remark 3 Since the power series $f_{A^{2}}(\lambda):=\sum_{n=0}^{\infty}\left|\alpha_{n}\right|^{2} \lambda^{n}$ is not easy to compute, we can provide some bounds for the quantity

$$
D_{f}(|\lambda|):=f_{A}^{2}(|\lambda|)-f_{A^{2}}\left(|\lambda|^{2}\right)
$$

where $|\lambda|<R$, as follows.

If $|\lambda|<1$ and $a_{\ell_{\infty}}:=\sup _{n \in \mathbb{N}}\left\{\left|a_{n}\right|\right\}<\infty$, then

$$
\begin{aligned}
K_{m} & \leq a_{\ell_{\infty}}^{2} \sum_{0 \leq j<n \leq m}|\lambda|^{n}|\lambda|^{j} \\
& =\frac{1}{2} a_{\ell_{\infty}}^{2}\left[\left(\sum_{n=0}^{m}|\lambda|^{n}\right)^{2}-\sum_{n=0}^{m}|\lambda|^{2 n}\right]
\end{aligned}
$$

and by taking $m \rightarrow \infty$ in this inequality we get

$$
D_{f}(|\lambda|) \leq \frac{1}{2} a_{\ell_{\infty}}^{2}\left[\left(\frac{1}{1-|\lambda|}\right)^{2}-\frac{1}{1-|\lambda|^{2}}\right]
$$

for $|\lambda|<1$.

If $|\lambda|<1$ and

$$
a_{\ell_{1}}:=\lim _{m \rightarrow \infty}\left[\left(\sum_{n=0}^{m}\left|\alpha_{n}\right|\right)^{2}-\sum_{n=0}^{m}\left|\alpha_{n}\right|^{2}\right]<\infty
$$

then

$$
\begin{aligned}
K_{m} & \leq \max _{n \in\{0, \ldots, m\}}|\lambda|^{2 n} \sum_{0 \leq j<n \leq m}\left|\alpha_{n}\right|\left|\alpha_{j}\right| \\
& \leq \frac{1}{2}\left[\left(\sum_{n=0}^{m}\left|\alpha_{n}\right|\right)^{2}-\sum_{n=0}^{m}\left|\alpha_{n}\right|^{2}\right]
\end{aligned}
$$

and by taking $m \rightarrow \infty$ in this inequality we get

$$
D_{f}(|\lambda|) \leq \frac{1}{2} a_{\ell_{1}}
$$

for $|\lambda|<1$.

If the series $\sum_{n=0}^{\infty}\left|\alpha_{n}\right|$ and $\sum_{n=0}^{\infty}\left|\alpha_{n}\right|^{2}$ are convergent, then

$$
D_{f}(|\lambda|) \leq \frac{1}{2}\left[\left(\sum_{n=0}^{\infty}\left|\alpha_{n}\right|\right)^{2}-\sum_{n=0}^{\infty}\left|\alpha_{n}\right|^{2}\right]
$$

for $|\lambda|<1$. 
If $|\lambda|<1, p, q>1$ with $\frac{1}{p}+\frac{1}{q}=1$, and

$$
a_{\ell_{q}}:=\lim _{m \rightarrow \infty}\left[\left(\sum_{n=0}^{m}\left|\alpha_{n}\right|^{q}\right)^{2}-\sum_{n=0}^{m}\left|\alpha_{n}\right|^{2 q}\right]<\infty
$$

then by Hölder's inequality we have

$$
\begin{aligned}
K_{m} \leq & \left.\sum_{0 \leq j<n \leq m}\left|\alpha_{n}\right|^{q}\left|\alpha_{j}\right|^{q}\right)^{1 / q}\left(\sum_{0 \leq j<n \leq m}|\lambda|^{p n}|\lambda|^{p j}\right)^{1 / p} \\
\leq & \left\{\frac{1}{2}\left[\left(\sum_{n=0}^{m}\left|\alpha_{n}\right|^{q}\right)^{2}-\sum_{n=0}^{m}\left|\alpha_{n}\right|^{2 q}\right]\right\}^{1 / q} \\
& \times\left\{\frac{1}{2}\left[\left(\sum_{n=0}^{m}|\lambda|^{p n}\right)^{2}-\sum_{n=0}^{m}|\lambda|^{2 p n}\right]\right\}^{1 / p}
\end{aligned}
$$

and by taking $m \rightarrow \infty$ in this inequality we get

$$
D_{f}(|\lambda|) \leq \frac{1}{2} a_{\ell_{q}}^{1 / q}\left[\left(\frac{1}{1-|\lambda|^{p}}\right)^{2}-\frac{1}{1-|\lambda|^{2 p}}\right]^{1 / p}
$$

for $|\lambda|<1$.

If the series $\sum_{n=0}^{\infty}\left|\alpha_{n}\right|^{q}$ and $\sum_{n=0}^{\infty}\left|\alpha_{n}\right|^{2 q}$ are convergent, then

$$
D_{f}(|\lambda|) \leq \frac{1}{2}\left[\left(\sum_{n=0}^{\infty}\left|\alpha_{n}\right|^{q}\right)^{2}-\sum_{n=0}^{\infty}\left|\alpha_{n}\right|^{2 q}\right]^{1 / p}\left[\left(\frac{1}{1-|\lambda|^{p}}\right)^{2}-\frac{1}{1-|\lambda|^{2 p}}\right]^{1 / p}
$$

for $|\lambda|<1$.

The following result also holds.

Theorem 3 Let $f(\lambda)=\sum_{n=0}^{\infty} \alpha_{n} \lambda^{n}$ be a function defined by power series with complex coefficients and convergent on the open disk $D(0, R) \subset \mathbb{C}, R>0$, and $x, y \in \mathcal{B}$ with $x y=y x$ and $\|x\|,\|y\|<1$.

If $p, q>1$ with $\frac{1}{p}+\frac{1}{q}=1$ and $\lambda \in \mathbb{C}$ with $|\lambda|,|\lambda|^{p},|\lambda|\|x\|,|\lambda|\|y\|<R$, then

$$
\begin{aligned}
\|\tilde{f}(\lambda \cdot 1) \tilde{f}(\lambda x y)-\tilde{f}(\lambda x) \tilde{f}(\lambda y)\| \\
\leq \frac{1}{2} \min \left\{\frac{\|x-1\|}{\left(1-\|x\|^{p}\right)^{1 / p}}, \frac{\|y-1\|}{\left(1-\|y\|^{p}\right)^{1 / p}}\right\} \\
\quad \times \varphi^{1 / q}\left[f_{A}^{2}\left(|\lambda|^{p}\right)-f_{A^{2}}\left(|\lambda|^{2 p}\right)\right]^{1 / p},
\end{aligned}
$$

where

$$
\varphi:=\lim _{m \rightarrow \infty} \sum_{n, j=0}^{m}\left|\alpha_{n}\right|\left|\alpha_{j}\right||n-j|
$$

is assumed to exist and be finite. 
Proof Using Hölder's inequality for $p, q>1$ with $\frac{1}{p}+\frac{1}{q}=1$ and (3.6), we have

$$
\begin{aligned}
L & \leq\|x-1\| \sum_{0 \leq j<n \leq m}\left|\alpha_{n} \| \alpha_{j}\right||\lambda|^{n}|\lambda|^{j}(n-j)^{1 / q}\left(\sum_{\ell=j}^{n-1}\|x\|^{\ell p}\right)^{1 / p} \\
& \leq\|x-1\|\left(\sum_{\ell=0}^{m-1}\|x\|^{\ell p}\right)^{1 / p} \sum_{0 \leq j<n \leq m}\left|\alpha_{n} \| \alpha_{j}\right||\lambda|^{n}|\lambda|^{j}(n-j)^{1 / q}
\end{aligned}
$$

for any $\lambda \in \mathbb{C}$ and $m \geq 1$.

Applying Hölder's inequality once more we have

$$
\begin{aligned}
\sum_{0 \leq j<n \leq m}\left|\alpha_{n}\right|\left|\alpha_{j}\right||\lambda|^{n}|\lambda|^{j}(n-j)^{1 / q} \\
\leq\left(\sum_{0 \leq j<n \leq m}\left|\alpha_{n}\right|\left|\alpha_{j}\right||\lambda|^{n}(n-j)\right)^{1 / q}\left(\sum_{0 \leq j<n \leq m}\left|\alpha_{n}\right|\left|\alpha_{j}\right||\lambda|^{p n}|\lambda|^{p j}\right)^{1 / p} \\
=\left(\frac{1}{2} \sum_{n, j=0}^{m}\left|\alpha_{n}\right|\left|\alpha_{j}\right||n-j|\right)^{1 / q} \\
\quad \times\left(\frac{1}{2}\left[\left(\sum_{n=0}^{m}\left|\alpha_{n}\right||\lambda|^{n p}\right)^{2}-\sum_{n=0}^{m}\left|\alpha_{n}\right|^{2}|\lambda|^{2 n p}\right]\right)^{1 / p} \\
=\frac{1}{2}\left(\sum_{n, j=0}^{m}\left|\alpha_{n}\right|\left|\alpha_{j}\right||n-j|\right)^{1 / q} \\
\quad \times\left[\left(\sum_{n=0}^{m}\left|\alpha_{n}\right||\lambda|^{n p}\right)^{2}-\sum_{n=0}^{m}\left|\alpha_{n}\right|^{2}|\lambda|^{2 n p}\right]^{1 / p}
\end{aligned}
$$

for any $\lambda \in \mathbb{C}$ and $m \geq 1$.

From (3.8) and (3.28) we get the inequality

$$
\begin{gathered}
\left\|\sum_{j=0}^{m} \alpha_{j} \lambda^{j} \sum_{n=0}^{m} \alpha_{n} \lambda^{n}(x y)^{n}-\sum_{j=0}^{m} \alpha_{j} \lambda^{j} x^{j} \sum_{n=0}^{m} \alpha_{n} \lambda^{n} y^{n}\right\| \\
\leq \frac{1}{2}\|x-1\|\left(\sum_{\ell=0}^{m-1}\|x\|^{\ell p}\right)^{1 / p}\left(\sum_{n, j=0}^{m}\left|\alpha_{n}\right|\left|\alpha_{j}\right||n-j|\right)^{1 / q} \\
\times\left[\left(\sum_{n=0}^{m}\left|\alpha_{n}\right||\lambda|^{n p}\right)^{2}-\sum_{n=0}^{m}\left|\alpha_{n}\right|^{2}|\lambda|^{2 n p}\right]^{1 / p}
\end{gathered}
$$

for any $\lambda \in \mathbb{C}$ and $m \geq 1$.

Since all the series whose partial sums are involved in (3.29) are convergent, then by letting $m \rightarrow \infty$ in (3.29) we deduce the desired inequality (3.25) for $x$. Due to the commutativity of $x$ with $y$, a similar result can be stated for $y$, and taking the minimum, we deduce the desired result. 
Remark 4 Observe that

$$
\left[f_{A}^{2}\left(|\lambda|^{p}\right)-f_{A^{2}}\left(|\lambda|^{2 p}\right)\right]^{1 / p}=D_{f}^{1 / p}\left(|\lambda|^{p}\right)
$$

and then further bounds for the inequality (3.25) may be provided by the use of Remark 3 . However the details are not mentioned here.

We can obtain a simpler upper bound for $\varphi$ as follows.

Using the Cauchy-Bunyakovsky-Schwarz inequality for double sums

$$
\sum_{n=0}^{m} \sum_{j=0}^{m} p_{i, j} a_{i, j} \leq\left(\sum_{n=0}^{m} \sum_{j=0}^{m} p_{i, j}\right)^{1 / 2}\left(\sum_{n=0}^{m} \sum_{j=0}^{m} p_{i, j} a_{i, j}^{2}\right)^{1 / 2}
$$

where $p_{i, j}, a_{i, j} \geq 0$ for $i, j \in\{0, \ldots, m\}$, we have

$$
\begin{aligned}
\sum_{n, j=0}^{m}\left|\alpha_{n}\right|\left|\alpha_{j}\right||n-j| & \leq\left(\sum_{n, j=0}^{m}\left|\alpha_{n}\right|\left|\alpha_{j}\right|\right)^{1 / 2}\left(\sum_{n, j=0}^{m}\left|\alpha_{n}\right|\left|\alpha_{j}\right||n-j|^{2}\right)^{1 / 2} \\
& =\sqrt{2} \sum_{n=0}^{m}\left|\alpha_{n}\right|\left[\sum_{n=0}^{m}\left|\alpha_{n}\right| \sum_{n=0}^{m} n^{2}\left|\alpha_{n}\right|-\left(\sum_{n=0}^{m} n\left|\alpha_{n}\right|\right)^{2}\right]^{1 / 2}
\end{aligned}
$$

for $m \geq 1$.

If the series $\sum_{n=0}^{\infty}\left|\alpha_{n}\right|$ is finite and $\psi$, defined by (3.2), is finite, then from (3.30) we have

$$
\varphi \leq \sqrt{2} \sum_{n=0}^{\infty}\left|\alpha_{n}\right| \psi
$$

We observe that, if the power series $f(\lambda)=\sum_{n=0}^{\infty} \alpha_{n} \lambda^{n}$ has the radius of convergence $R>1$, then $\psi$ is finite and

$$
\psi=\left\{f_{A}(1)\left[f_{A}^{\prime}(1)+f_{A}^{\prime \prime}(1)\right]-\left[f_{A}^{\prime}(1)\right]^{2}\right\}^{1 / 2}
$$

We have from (3.31) the inequality

$$
\varphi \leq \sqrt{2} f_{A}(1)\left\{f_{A}(1)\left[f_{A}^{\prime}(1)+f_{A}^{\prime \prime}(1)\right]-\left[f_{A}^{\prime}(1)\right]^{2}\right\}^{1 / 2}
$$

\section{Some examples}

As some natural examples that are useful for applications, we can point out that, if

$$
\begin{aligned}
& f(\lambda)=\sum_{n=1}^{\infty} \frac{(-1)^{n}}{n} \lambda^{n}=\ln \frac{1}{1+\lambda}, \quad \lambda \in D(0,1) ; \\
& g(\lambda)=\sum_{n=0}^{\infty} \frac{(-1)^{n}}{(2 n) !} \lambda^{2 n}=\cos \lambda, \quad \lambda \in \mathbb{C} ; \\
& h(\lambda)=\sum_{n=0}^{\infty} \frac{(-1)^{n}}{(2 n+1) !} \lambda^{2 n+1}=\sin \lambda, \quad \lambda \in \mathbb{C} ; \\
& l(\lambda)=\sum_{n=0}^{\infty}(-1)^{n} \lambda^{n}=\frac{1}{1+\lambda}, \quad \lambda \in D(0,1),
\end{aligned}
$$


then the corresponding functions constructed by the use of the absolute values of the coefficients are

$$
\begin{aligned}
& f_{A}(\lambda)=\sum_{n=1}^{\infty} \frac{1}{n} \lambda^{n}=\ln \frac{1}{1-\lambda}, \quad \lambda \in D(0,1) ; \\
& g_{A}(\lambda)=\sum_{n=0}^{\infty} \frac{1}{(2 n) !} \lambda^{2 n}=\cosh \lambda, \quad \lambda \in \mathbb{C} ; \\
& h_{A}(\lambda)=\sum_{n=0}^{\infty} \frac{1}{(2 n+1) !} \lambda^{2 n+1}=\sinh \lambda, \quad \lambda \in \mathbb{C} ; \\
& l_{A}(\lambda)=\sum_{n=0}^{\infty} \lambda^{n}=\frac{1}{1-\lambda}, \quad \lambda \in D(0,1) .
\end{aligned}
$$

Other important examples of functions as power series representations with nonnegative coefficients are

$$
\begin{aligned}
& \exp (\lambda)=\sum_{n=0}^{\infty} \frac{1}{n !} \lambda^{n}, \quad \lambda \in \mathbb{C}, \\
& \frac{1}{2} \ln \left(\frac{1+\lambda}{1-\lambda}\right)=\sum_{n=1}^{\infty} \frac{1}{2 n-1} \lambda^{2 n-1}, \quad \lambda \in D(0,1) \\
& \sin ^{-1}(\lambda)=\sum_{n=0}^{\infty} \frac{\Gamma\left(n+\frac{1}{2}\right)}{\sqrt{\pi}(2 n+1) n !} \lambda^{2 n+1}, \quad \lambda \in D(0,1) \\
& \tanh ^{-1}(\lambda)=\sum_{n=1}^{\infty} \frac{1}{2 n-1} \lambda^{2 n-1}, \quad \lambda \in D(0,1) ; \\
& { }_{2} F_{1}(\alpha, \beta, \gamma, \lambda)=\sum_{n=0}^{\infty} \frac{\Gamma(n+\alpha) \Gamma(n+\beta) \Gamma(\gamma)}{n ! \Gamma(\alpha) \Gamma(\beta) \Gamma(n+\gamma)} \lambda^{n}, \quad \alpha, \beta, \gamma>0, \lambda \in D(0,1)
\end{aligned}
$$

where $\Gamma$ is the Gamma function.

If we apply the inequality (3.13) to the exponential function, then we have

$$
\|\exp [\lambda(1+x y)]-\exp [\lambda(x+y)]\| \leq \sqrt{2} e \min \{\|x-1\|,\|y-1\|\} \exp \left(|\lambda|^{2}\right)
$$

for any $x, y \in \mathcal{B}$ with $x y=y x,\|x\|,\|y\|<1$, and $\lambda \in \mathbb{C}$.

If we take $y=-x$ in (4.4), then we get

$$
\left\|\exp \left[\lambda\left(1-x^{2}\right)\right]-1\right\| \leq \sqrt{2} e \min \{\|x-1\|,\|x+1\|\} \exp \left(|\lambda|^{2}\right)
$$

for any $x \in \mathcal{B}$ with $\|x\|<1$ and $\lambda \in \mathbb{C}$.

If we apply the inequality (3.3) for the exponential functions we also have

$$
\begin{aligned}
& \|\exp [\lambda(1+x y)]-\exp [\lambda(x+y)]\| \\
& \quad \leq \sqrt{2} \min \{\|x-1\|,\|y-1\|\}|\lambda|^{1 / 2} \exp (2|\lambda|),
\end{aligned}
$$

for any $x, y \in \mathcal{B}$ with $x y=y x,\|x\|,\|y\|<1$, and $\lambda \in \mathbb{C}$. 
If we take $y=-x$ in (4.6), then we get

$$
\left\|\exp \left[\lambda\left(1-x^{2}\right)\right]-1\right\| \leq \sqrt{2} \min \{\|x-1\|,\|x+1\|\}|\lambda|^{1 / 2} \exp (2|\lambda|)
$$

Now, consider the function $f(\lambda):=\sum_{n=0}^{\infty} \lambda^{n}=\frac{1}{1-\lambda}, \lambda \in D(0,1)$. If we apply the inequality (3.3) for this function, then we get the result

$$
\begin{aligned}
& \left\|(1-\lambda)^{-1}(1-\lambda x y)^{-1}-(1-\lambda x)^{-1}(1-\lambda y)^{-1}\right\| \\
& \quad \leq \sqrt{2} \min \{\|x-1\|,\|y-1\|\}|\lambda|^{1 / 2}(1-|\lambda|)^{-3}
\end{aligned}
$$

for any $x, y \in \mathcal{B}$ with $x y=y x,\|x\|,\|y\|<1$, and $\lambda \in \mathbb{C}$ with $|\lambda|<1$.

We have in particular the inequalities

$$
\left\|(1-\lambda)^{-1}\left(1-\lambda x^{2}\right)^{-1}-(1-\lambda x)^{-2}\right\| \leq \sqrt{2}\|x-1\||\lambda|^{1 / 2}(1-|\lambda|)^{-3}
$$

and

$$
\begin{aligned}
& \left\|(1-\lambda)^{-1}\left(1+\lambda x^{2}\right)^{-1}-\left(1-\lambda^{2} x^{2}\right)^{-1}\right\| \\
& \quad \leq \sqrt{2} \min \{\|x-1\|,\|x+1\|\}|\lambda|^{1 / 2}(1-|\lambda|)^{-3}
\end{aligned}
$$

for any $x \in \mathcal{B}$ with $\|x\|<1$ and $\lambda \in \mathbb{C}$ with $|\lambda|<1$.

Now, if we take $\lambda=\frac{1}{\gamma}$ with $|\gamma|>1$ then we get from (4.8) the inequality

$$
\begin{aligned}
& \left\|\gamma^{2}(\gamma-1)^{-1}(\gamma-x y)^{-1}-\gamma^{2}(\gamma-x)^{-1}(\gamma-y)^{-1}\right\| \\
& \quad \leq \sqrt{2} \min \{\|x-1\|,\|y-1\|\}|\gamma|^{-1 / 2}(|\gamma|-1)^{-3}|\gamma|^{3},
\end{aligned}
$$

which is equivalent with

$$
\begin{aligned}
& \left\|(\gamma-1)^{-1}(\gamma-x y)^{-1}-(\gamma-x)^{-1}(\gamma-y)^{-1}\right\| \\
& \quad \leq \sqrt{2} \min \{\|x-1\|,\|y-1\|\}|\gamma|^{1 / 2}(|\gamma|-1)^{-3}
\end{aligned}
$$

for any $x, y \in \mathcal{B}$ with $x y=y x,\|x\|,\|y\|<1$, and $\gamma \in \mathbb{C}$ with $|\gamma|>1$.

If we use the resolvent function notation, then we have the following inequality:

$$
\begin{aligned}
& \left\|(\gamma-1)^{-1} R_{x y}(\gamma)-R_{x}(\gamma) R_{y}(\gamma)\right\| \\
& \quad \leq \sqrt{2} \min \{\|x-1\|,\|y-1\|\}|\gamma|^{1 / 2}(|\gamma|-1)^{-3}
\end{aligned}
$$

for any $x, y \in \mathcal{B}$ with $x y=y x,\|x\|,\|y\|<1$, and $\gamma \in \mathbb{C}$ with $|\gamma|>1$.

In particular, we have

$$
\left\|(\gamma-1)^{-1} R_{x^{2}}(\gamma)-R_{x}^{2}(\gamma)\right\| \leq \sqrt{2}\|x-1\||\gamma|^{1 / 2}(|\gamma|-1)^{-3}
$$

for any $x \in \mathcal{B}$ with $\|x\|<1$ and $\gamma \in \mathbb{C}$ with $|\gamma|>1$. 
Remark 5 Similar inequalities may be stated for the other power series mentioned at the beginning of this paragraph. However, the details are not presented here.

\section{Competing interests}

The authors declare that they have no competing interests.

\section{Authors' contributions}

All authors contributed equally and significantly in writing this article. All authors read and approved the final manuscript.

\section{Author details}

1 Mathematics, School of Engineering \& Science, Victoria University, PO Box 14428, Melbourne City, MC 8001, Australia ${ }^{2}$ School of Computational \& Applied Mathematics, University of the Witwatersrand, Private Bag 3, Johannesburg, 2050, South Africa. ${ }^{3}$ Mathematics and Statistics, Banat University of Agricultural Sciences and Veterinary Medicine Timişoara, 119 Calea Aradului, Timişoara, 300645, România. ${ }^{4}$ Department of Mathematics, West University of Timişoara, B-dul V. Pârvan 4, Timişoara, 1900, România.

\section{Acknowledgements}

The authors would like to thank the anonymous referees for their valuable comments that have been implemented in the final version of the paper.

Received: 20 February 2014 Accepted: 27 June 2014 Published: 18 August 2014

\section{References}

1. Grüss, G: Über das Maximum des absoluten Betrages von $\frac{1}{b-a} \int_{a}^{b} f(x) g(x) d x-\frac{1}{(b-a)^{2}} \int_{a}^{b} f(x) d x \int_{a}^{b} g(x) d x$. Math. Z. 39, 215-226 (1935)

2. Chebyshev, PL: Sur les expressions approximatives des intégrals définies par les autres prises entre les mêmes limites Proc. Math. Soc. Charkov 2, 93-98 (1882)

3. Ostrowski, AM: On an integral inequality. Aequ. Math. 4, 358-373 (1970)

4. Lupaş, A: The best constant in an integral inequality. Mathematica 15(38)(2), 219-222 (1973)

5. Cerone, P, Dragomir, SS: New bounds for the Čebyšev functional. Appl. Math. Lett. 18, 603-611 (2005)

6. Cheng, X-L, Sun, J: Note on the perturbed trapezoid inequality. J. Inequal. Pure Appl. Math. 3(2), 29 (2002). http://www.emis.de/journals/JIPAM/article181.html?sid=181

7. Cerone, P, Dragomir, SS: A refinement of the Grüss inequality and applications. Tamkang J. Math. 38(1), 37-49 (2007). Preprint, RGMIA Res. Rep. Coll. 5(2), Art. 14 (2002). http://rgmia.org/papers/v5n2/RGIApp.pdf

8. Alomari, MW: Some Grüss type inequalities for Riemann-Stieltjes integral and applications. Acta Math. Univ. Comen. 81(2), 211-220 (2012)

9. Dragomir, SS: Some inequalities for Riemann-Stieltjes integral and applications. In: Rubinov, A (ed.) Optimisation and Related Topics, pp. 197-235. Kluwer Academic, Dordrecht (2000)

10. Dragomir, SS: Sharp bounds of Čebyšev functional for Stieltjes integrals and applications. Bull. Aust. Math. Soc. 67(2), 257-266 (2003)

11. Dragomir, SS: New estimates of the Čebyšev functional for Stieltjes integrals and applications. J. Korean Math. Soc. 41(2), 249-264 (2004)

12. Dragomir, SS: Čebyšev's type inequalities for functions of selfadjoint operators in Hilbert spaces. Linear Multilinear Algebra 58(7-8), 805-814 (2010)

13. Dragomir, SS: Inequalities for the Čebyšev functional of two functions of selfadjoint operators in Hilbert spaces. Aust. J. Math. Anal. Appl. 6(1), 1-58 (2009)

14. Dragomir, SS: Some inequalities for power series of selfadjoint operators in Hilbert spaces via reverses of the Schwarz inequality. Integral Transforms Spec. Funct. 20(9-10), 757-767 (2009)

15. Gavrea, B: Improvement of some inequalities of Chebysev-Grüss type. Comput. Math. Appl. 64(6), 2003-2010 (2012)

16. Li, X, Mohapatra, RN, Rodriguez, RS: Grüss-type inequalities. J. Math. Anal. Appl. 267(2), 434-443 (2002)

17. Mercer, AM: An improvement of the Grüss inequality. J. Inequal. Pure Appl. Math. 6(4), Article ID 93 (2005)

18. Otachel, Z: Grüss type inequalities in normed spaces. J. Math. Anal. Appl. 393(1), 14-24 (2012)

19. Pachpatte, BG: On Grüss like integral inequalities via Pompeiu's mean value theorem. J. Inequal. Pure Appl. Math. 6(3), Article ID 82 (2005)

20. Dragomir, SS, Pečarić, J, Sándor, J: The Chebyshev inequality in pre-Hilbertian spaces. II. In: Proceedings of the Third Symposium of Mathematics and Its Applications, Timişoara, 1989, pp. 75-78. Rom. Acad, Timişoara (1990). MR1266442 (94m:46033)

21. Dragomir, SS, Sándor, J: The Chebyshev inequality in pre-Hilbertian spaces. I. In: Proceedings of the Second Symposium of Mathematics and Its Applications, Timişoara, 1987, pp. 61-64. Res. Centre, Acad. SR, Romania, Timişoara (1988). MR1006000 (90k:46048)

22. Liu, Z: Refinement of an inequality of Grüss type for Riemann-Stieltjes integral. Soochow J. Math. 30(4), 483-489 (2004)

23. Cohen, JE: Chebyshev and Grüss inequalities for real rectangular matrices. Linear Algebra Appl. 447, 133-138 (2014)

24. Jocić, DR, Krtinić, D, Moslehian, MS: Landau and Grüss type inequalities for inner product type integral transformers in norm ideals. Math. Inequal. Appl. 16(1), 109-125 (2013)

25. Matharu, JS, Aujla, JS: Hadamard product versions of the Chebyshev and Kantorovich inequalities. J. Inequal. Pure Appl. Math. 10(2), 51 (2009)

26. Moslehian, MS, Bakherad, M: Chebyshev type inequalities for Hilbert space operators. J. Math. Anal. Appl. (2014). doi:10.1016/j.jmaa.2014.05.078 
27. Dragomir, SS: Operator Inequalities of the Jensen, Čebyšev and Grüss Type. Springer Briefs in Mathematics. Springer, New York (2012). ISBN 978-1-4614-1520-6

28. Dragomir, SS: Operator Inequalities of Ostrowski and Trapezoidal Type. Springer Briefs in Mathematics. Springer, New York (2012). ISBN 978-1-4614-1778-1

29. Furuta, T, Mićić Hot, J, Pečarić, J, Seo, Y: Mond-Pečarić Method in Operator Inequalities. Inequalities for Bounded Selfadjoint Operators on a Hilbert Space. Element, Zagreb (2005)

doi:10.1186/1029-242X-2014-294

Cite this article as: Dragomir et al.: Norm inequalities of Čebyšev type for power series in Banach algebras. Journal of Inequalities and Applications 2014 2014:294.

Submit your manuscript to a SpringerOpen ${ }^{\odot}$ journal and benefit from:

- Convenient online submission

- Rigorous peer review

- Immediate publication on acceptance

- Open access: articles freely available online

- High visibility within the field

- Retaining the copyright to your article

Submit your next manuscript at $\boldsymbol{s p r i n g e r o p e n . c o m ~}$ 